\title{
The Effect Of Diet Composition On Weight Gain in Obese, Type 2 Diabetes Patients Receiving Intensive Insulin Therapy
}

\author{
Roland Villareal, VA Southern Nevada Healthcare System, Las Vegas, Nevada
}

\begin{abstract}
This study was conducted to contribute to the limiting existing body of literature about diet and prevention of weight gain when administering intensive insulin therapy. The effects of a highmonounsaturated fatty acid (MUFA) diet compared with a conventional diabetic diet have not been studied in insulin treated patients. A growing body of evidence assessed that diets rich in high-MUFA foods had similar glycemic results, as do low-fat, high carbohydrate diets. However, a high-MUFA diet did not raise triglycerides as suspected. Ros (2003) stated that high-MUFA energy controlled diets do not promote weight gain and are more acceptable for weight loss and/or maintenance. A MUFA diet can be used as an alternate to the conventional American Diabetic Association (ADA) diet when managing obese type 2 diabetes patients treated with intensive insulin therapy. Dietary restriction to 1600 calories in diabetes patients on intensive insulin therapy decreased the AIC value by 1.3 points in the ADA group and 1.5 points in the MUFA group without weight gain and without additional insulin required. In conclusion the total calorie count was more important for preventing weight gain and reducing the A1C in patients on intensive insulin therapy than was dietary composition.
\end{abstract}

Keywords: High-MUFA Diet; Intensive Insulin Therapy

\section{INTRODUCTION}

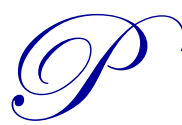

atients starting insulin therapy gain weight. Some patients gain more weight by eating additional food or snacks to avoid hypoglycemia (Wijenaike, 2004). The purpose of the study was to determine what effect a high-monounsaturated fatty acid diet has on weight gain, metabolic outcomes and hypoglycemia in obese type 2 diabetes patients receiving intensive insulin therapy. A challenging dimension in the management of diabetes is nutrition. Glycemic control and maintaining a reasonable body weight is a realistic goal. In obese, insulin resistant, type 2 diabetes patients who are receiving intensive insulin therapy a dietary regimen of reduced calories is essential for weight loss or maintenance. According to Mayfield and White (2004), weight gain can be modified through moderate exercise and reduced calories. There is much controversy on what diet is best for diabetic patients and more research is needed in this area. There were no studies in the literature that examined the effects of a high-MUFA diet in obese type 2 diabetes patients starting intensive insulin therapy. This group of insulin treated diabetes patient's remains unique to study and will contribute meaningful information to practitioners in the field.

\section{LITERATURE REVIEW}

The effects of a high-MUFA diet compared with a conventional diabetic diet have not been studied in insulin treated patients. A growing body of evidence assessed that diets rich in high-MUFA foods had similar Glycemic results, as do low-fat, high carbohydrate diets. According to Ros (2003), high-MUFA diets tend to lower triglycerides and raise high density lipoproteins (HDL). Ros determined that high-MUFA energy controlled diets do not promote weight gain and are more acceptable for weight loss. Flynn and Colquhoun (2006) reported that weight loss programs based on a Mediterranean type diet (high-MUFA) were very effective in both short term and long 
term weight loss. McManus et al. (2001) assessed long term compliance with the diet and improvements in weight loss in higher fat diets than lower fat diets. Ros (2003) further identified a positive relationship between higher palatability and compliance with a high-MUFA diet. Kris-Etherton (1999) identified foods rich in oleic acid oils and fats provided the cardiovascular benefits; however, nutrition experts remain reluctant in prescribing a highMUFA diet as an alternative to the typical ADA diet. The novelty of this research study is based on an assessment of diabetes clinical outcomes in a unique population of obese type 2 diabetes patients on intensive insulin therapy.

The prevalence of obesity in America continues to rise (Flegal et al., 2002) and obese, type 2 diabetes patients can expect more weight gain associated with intensive insulin therapy. Obesity is a major contributor to type 2 diabetes (Hu, Lindstrom, Valle \& Eriksson et al., 2004). Early reports by Reaven (1988) referred the cluster of abdominal obesity, hypertriglyceridemia, low HDL cholesterol, hyperinsulinemia, glucose intolerance and hypertension as the "Syndrome X". Today, this is called metabolic syndrome or insulin resistance (Kopeland \& Grace, 2004; Bonow \& Eckel, 2003). Obese individuals tend to live sedentary lifestyle, which leads to an increase risk of developing diabetes. Marks (2004) reported less than one-third of U.S. adults participate in leisure-time physical activity and $40 \%$ of adults did no physical activity at all. In their study of physical activity, BMI and type 2 diabetes, $\mathrm{Hu}$ et al. assessed that physical activity independently and significantly reduced the risk of type 2 diabetes.

The objective of intensive insulin therapy or multiple daily insulin injections is to achieve optimal glycemic control. The American Diabetes Association standard of recommends tight glucose control and patients who fail oral anti-diabetic medication require insulin treatment. The United Kingdom Prospective Diabetes Study (UKPDS) and the Diabetes Control and Complications Trial (DCCT) recommended the HgAlc treatment goal of 7\%. While insulin therapy can improve glycemic control and prevent long-term complications in type 2 diabetes, adverse effects are common. The two major adverse effects of intensive insulin therapy are hypoglycemia and weight gain.

\section{OBJECTIVE}

This paper will examine whether obese type 2 diabetes patients starting intensive insulin therapy experience less weight gain and/or maintain body weight by consuming a high-monounsaturated fatty acid (MUFA) diet compared to patients consuming a conventional diabetic (ADA) diet. Secondary objectives of the study will determine whether the effect of a high-MUFA diet will lead to: (1) better glycemic control, (2) better lipid control, (3) lower blood pressure levels, (4) require less insulin, and (5) experience fewer hypoglycemia events.

\section{RESEARCH DESIGN AND METHODS}

This research was a randomized, open label, intervention study. The sample included 76 male type 2 diabetic patients starting intensive insulin therapy. The methodology was a 35-week, study conducted in an outpatient diabetes clinic at the Veterans Affairs Southern Nevada Healthcare System in Las Vegas, Nevada. Seventy-six male veterans with type 2 diabetes and a BMI greater than 30 were randomized to a 1600 calorie ADA $\operatorname{diet}(\mathrm{N}=39)$ or a 1600 calorie MUFA $\operatorname{diet}(\mathrm{N}=37)$. All participants were newly started on intensive insulin therapy. Data analysis used the SPSS software 16.0 version. Descriptive statistics, the independent $t$-test, relative risk, and Pearson's chi-square measures were utilized to analyze quantitative data. Subjects met the following characteristics based on the inclusion and exclusion criteria: $\mathrm{BMI}>30, \mathrm{HgA} 1 \mathrm{c}>7.0 \%$, on intensive insulin therapy and were physically inactive. Subjects with unstable cardiovascular, renal, respiratory, gastrointestinal disease or malignancy were excluded. Recruitment and research of subjects was conducted in accordance with HIPAA guidelines and all subjects gave informed written consent. Selection of the appropriate sample size was based on similar past studies. The outcome variable was the amount of weight gained on intensive insulin therapy after treatment over six months and a two month follow up assessment. The number of patients required in each group at an alpha (two tailed) $=0.05$ and power $=0.90$ was 33 . Previous studies reported a mean weight gain of 10 pounds $(5 \mathrm{~kg}$.) with a standard deviation of $0.6 \mathrm{~kg}$. An isocaloric diet of 1600 calories per day was suggested according to ADA recommendations for obese men and women who want to lose weight. Diet randomization was performed within one week of consent. The control group consisted of patients consuming a conventional diabetic diet: 1600 calories per day with $20 \%$ energy from total fats (10\% energy saturated fat and $10 \%$ energy other fat); $65 \%$ energy from carbohydrates; and $15 \%$ energy from proteins. The treatment group consisted of patients consuming a highMUFA diet: 1600 calories per day, with $40 \%$ energy from total fats (energy $10 \%$ saturated fat, $10 \%$ other fat, $20 \%$ 
MUFA); $45 \%$ energy from carbohydrates; and 15\% energy from proteins. Data was collected on anthropometric measures, biochemical measures and a questionnaire by the person doing the study. Body weight, height and BMI were calculated. Measures in waist circumference, hip circumference and waist-to-hip ratio were also calculated. Participant's blood pressure was checked manually and the average of three readings for systolic and diastolic pressure was obtained. Participants were sent to the medical laboratory for blood work. Biochemical measures taken in the laboratory included fasting plasma glucose, Hemoglobin Alc (HgAlc), and the lipoproteins of TCHO, LDL, HDL, and TG. Documentation of blood sugars monitored in telehealth records or patient blood sugar diaries was assessed. The endocrinologist reviewed Self-Monitoring Blood Glucose results and made adjustments to the insulin dose as required to achieve Glycemic control.

\section{FINDINGS}

Descriptive analysis was collected on 76 participants. Males represented $100 \%$ of the sample size. All participants were overweight, diagnosed with type 2 diabetes and newly starting intensive insulin therapy. The study was designed to recruit ages 20 to 80 years. The average age of participants was 60 years with a minimum age of 39 , and a maximum age of 77 . The mean comparison of study participants body measurements and laboratory values at baseline and at the end of the study (35 weeks) are presented in table 1 . The mean body weight measurement was $111 \mathrm{~kg}(\mathrm{SD}=21)$ at baseline and $110 \mathrm{~kg}(\mathrm{SD}=22)$ at 35 weeks in the ADA group. Participants in this group lost $1 \mathrm{~kg}$ on the ADA diet. The mean body weight measurement was $120 \mathrm{~kg}(\mathrm{SD}=23)$ at baseline and $119 \mathrm{~kg}(\mathrm{SD}=23)$ at 35 weeks in the MUFA group. Participants in this group lost $1 \mathrm{~kg}$ on the MUFA diet. In both groups, there was a weight loss of $1 \mathrm{~kg}$, which means that both groups loss a mean of 2.2 pounds.

Table 1. Mean Comparison of Participants Body Measurements and Laboratory Values at Baseline (week 0) and at Follow-up (week 35)

\begin{tabular}{|c|c|c|c|c|}
\hline & \multicolumn{2}{|c|}{ ADA $(N=39)$} & \multicolumn{2}{|c|}{ MUFA $(\mathbf{N}=\mathbf{3 7})$} \\
\hline & Week 0 & Week 35 & Week 0 & Week 35 \\
\hline Characteristic & $\mathrm{M}(\mathrm{SD})$ & $\mathrm{M}(\mathrm{SD})$ & $\mathrm{M}(\mathrm{SD})$ & $\mathrm{M}(\mathrm{SD})$ \\
\hline Weight (kg) & $111(21)$ & $110(22)$ & $120(23)$ & $119(23)$ \\
\hline BMI (kg/m2) & $36(5.2)$ & $35.8(5.5)$ & $39(6.3)$ & $38.3(6.3)$ \\
\hline WHR & $1.01(.06)$ & $1.01(0.06)$ & $1.01(.06)$ & $1.01(0.06)$ \\
\hline HgAlc (\%) & $9.3(1.5)$ & $8.0(1.7)$ & $9.6(1.8)$ & $8.1(1.4)$ \\
\hline FPG $(\mathrm{mg} / \mathrm{dl})$ & $192(76.8)$ & $140(78.1)$ & $192(86.3)$ & $150(56)$ \\
\hline TCHO (mg/dl) & $185(48.5)$ & $167(41.1)$ & $167(57.3)$ & $158(43)$ \\
\hline HDL (mg/dl) & $34(10.2)$ & $36(10.8)$ & $29.5(15.23)$ & $32(6.7)$ \\
\hline $\mathrm{LDL}(\mathrm{mg} / \mathrm{dl})$ & $104(38.1)$ & $101(35)$ & $73.8(48.30)$ & $96(43)$ \\
\hline $\mathrm{TG}(\mathrm{mg} / \mathrm{dl})$ & $240(228)$ & $154(92)$ & $312(451)$ & $190(151)$ \\
\hline $\mathrm{SBP}(\mathrm{mmHg})$ & $133(15)$ & $129(12)$ & $134(12)$ & $127(12)$ \\
\hline DBP (mmHg) & $76(10)$ & $74(10)$ & $78(7)$ & $75(9)$ \\
\hline Insulin (IU) & $99(63)$ & $111(63)$ & $106(57)$ & $118(68)$ \\
\hline
\end{tabular}

T-tests between the independent variable of diet composition, 1600 calorie ADA and 1600 calorie highMUFA diet, and the dependent variables of weight, BMI, WHR, HgA1c, TCHO, HDL, LDL, TG, SBP, DBP, and insulin level were run and are presented in table 2. The independent $t$-test revealed no association between the effects of an ADA diet $(\mathrm{M}=-.30, \mathrm{SD}=6.6)$ and a high-MUFA diet $(\mathrm{M}=-.57, \mathrm{SD}=5.6)$ on weight gain $(\mathrm{t}(.19), \mathrm{p}>$ .05 ). The null hypothesis was accepted that there is no association between the effects of diet composition on weight gain in obese, type 2 diabetes patients receiving intensive insulin therapy; and the alternate hypothesis that there is an association was rejected. The null hypothesis was accepted that there is no association between the effects of diet composition on weight gain in obese, type 2 diabetes patients receiving intensive insulin therapy; and the alternate hypothesis that there is an association was rejected. As previously stated, the initial mean weight for the ADA group was of $111 \mathrm{~kg}$, while the initial mean weight for the MUFA group was $120 \mathrm{~kg}$. At 35 weeks, the mean weight for the ADA group was $110 \mathrm{~kg}$ and the mean weight for the MUFA group was $119 \mathrm{~kg}$. Thus, at 35 weeks, the MUFA group continued to be $9.0 \mathrm{~kg}$ more than the ADA group in spite of weight loss in both groups. As a note of interest, there was no additional weight gain for either group. 
Table 2. Comparison of T-test Between Diet Composition and Participant Characteristics at End of Follow-up

\begin{tabular}{lcc|cc}
\hline & ADA & MUFA & & $\boldsymbol{t}$ \\
\hline \multicolumn{1}{c}{ Characteristic } & M (SD) & M (SD) & .19 & .85 \\
\hline Weight kg $(\mathrm{n}=72)$ & $-.30(6.6)$ & $-.57(5.6)$ & -.27 & .79 \\
BMI kg/m2 (n=72) & $-.36(2.5)$ & $-.22(1.9)$ & -.88 & .38 \\
WHR (n=72) & $-.01(.06)$ & $-.002(.05)$ & .91 & .36 \\
A1c \% $(\mathrm{n}=71)$ & $-1.21(1.8)$ & $-1.61(2.0)$ & -.26 & .79 \\
FPG mg/dl $(\mathrm{n}=71)$ & $-50.4(102)$ & $-44.12(99)$ & -1.36 & .18 \\
TCHO mg/dl $(\mathrm{n}=70)$ & $-18.13(44)$ & $-2.58(52)$ & 1.11 & .27 \\
HDL mg/dl $(\mathrm{n}=67)$ & $1.08(7.3)$ & $-7.1(5.7)$ & -.26 & .80 \\
LDL mg/dl $(\mathrm{n}=64)$ & $-1.7(35.9)$ & $-.79(40.2)$ & 1.06 & .29 \\
TG mg/dl $(\mathrm{n}=70)$ & $-67.3(168)$ & $-138(363)$ & 1.89 & .06 \\
SBP mmHg $(\mathrm{n}=72)$ & $2.11(16.6)$ & $-4.74(14)$ & .76 & .45 \\
DBP mmHg $(\mathrm{n}=72)$ & $-.81(11)$ & $-2.6(8.8)$ & 1.64 & .11 \\
Insulin IU $(\mathrm{n}=72)$ & $13.6(29)$ & $1.91(32)$ & & \\
\hline
\end{tabular}

The relative risk statistic was used to compare the risk of developing hypoglycemia in people consuming a MUFA diet as compared to people who are not receiving the treatment but rather consuming the established ADA diet. Table 3, provides a contingency table that describes the type of diet consumed and number of hypoglycemia events experienced. In the ADA group, 29 (78.4\%) reported experiencing hypoglycemia compared to $26(74.3 \%)$ in the MUFA group.

Table 3. Contingency Table for Type of Diet and Hypoglycemia Events

\begin{tabular}{|c|c|c|c|}
\hline \multirow{2}{*}{ Group } & \multicolumn{2}{|c|}{ Hypoglycemia } & \multirow{2}{*}{ Total } \\
\hline & Yes & No & \\
\hline $\begin{array}{l}\text { ADA Diet Count (control) } \\
\% \text { within Type of Diet }\end{array}$ & $\begin{array}{c}29 \\
78.4 \%\end{array}$ & $\begin{array}{c}8 \\
21.6 \%\end{array}$ & $\begin{array}{c}37 \\
100 \%\end{array}$ \\
\hline $\begin{array}{r}\text { MUFA Diet Count (treatment) } \\
\% \text { within Type of Diet }\end{array}$ & $\begin{array}{c}26 \\
74.3 \%\end{array}$ & $\begin{array}{c}9 \\
25.7 \%\end{array}$ & $\begin{array}{c}35 \\
100 \%\end{array}$ \\
\hline $\begin{array}{l}\text { Total } \\
\end{array}$ & $\begin{array}{c}55 \\
76.4 \%\end{array}$ & $\begin{array}{c}17 \\
23.6 \%\end{array}$ & $\begin{array}{c}72 \\
100 \%\end{array}$ \\
\hline
\end{tabular}

Those who reported no hypoglycemia events were $8.0(21.6 \%)$ in the ADA group and $9.0(25.7 \%)$ in the MUFA group. A total of $55(76.4 \%)$ reported experiencing hypoglycemia and a total of $17(23.6 \%)$ of 72 participants reported no hypoglycemia events. The relative risk for hypoglycemia was calculated based on the data presented in table 4. The risk for hypoglycemia in participants consuming an ADA diet was 1.06 and was not significant, $\mathrm{RR}=1.05 ; 95 \% \mathrm{CI}=(.815,1.366)$. The value of 1.0 was between the lower and higher $\mathrm{CI}$, thus the result is not statistically significant. Pearson chi-square test of independence was used to evaluate the categorical variables of diet composition and the dichotomous variable of hypoglycemia events. The chi-square test identified that there is no association between an ADA diet and a MUFA diet and its effects on hypoglycemia, $[\chi 2(1, N=72)$ $=.167, \mathrm{p}=.683]$, in obese, type 2 diabetes patients receiving intensive insulin therapy. The null hypothesis was accepted and the alternate hypothesis was rejected.

Table 4. Risk Estimate Table for Type of Diet and Hypoglycemia Events

\begin{tabular}{lccc}
\hline & \multirow{2}{*}{ Value } & 95\% Confidence Interval \\
\cline { 2 - 3 } & & Lower & Upper \\
\hline Odds Ratio for Type of Diet (ADA/MUFA & 1.255 & .422 & 3.731 \\
For Cohort low Blood Sugar = yes & 1.055 & .815 & 1.366 \\
For Cohort low blood sugar = no & .841 & .366 & 1.934 \\
N of Valid Cases & 72 & & \\
\hline
\end{tabular}

\section{CONCLUSIONS}

This research study observed whether obese (BMI > 30), type 2 diabetes patients starting intensive insulin therapy can control and/or lose weight, improve glycemia, improve blood lipids and blood pressure, and experience lower hypoglycemia events by adhering to a high-MUFA diet compared to a conventional diabetic diet. T-tests 
revealed no association $(\mathrm{p}>.05)$ between the effects of diet composition on weight gain $(\mathrm{p}=.90)$, BMI $(\mathrm{p}=.80)$, WHR $(\mathrm{p}=.38)$, Alc $(\mathrm{p}=.36)$, FPG $(\mathrm{p}=.80)$, TCHOL $(\mathrm{p}=.18)$, HDL $(\mathrm{p}=.27)$, LDL $(\mathrm{p}=.80)$, TG $(\mathrm{p}=.30)$, SBP $(\mathrm{p}=.06)$, DBP $(\mathrm{p}=.45)$ and insulin dose $(\mathrm{p}=.11)$. Pearson's chi square for independence identified no association $(\mathrm{p}=.683)$ between the effects of diet composition on hypoglycemia events. This study was conducted to contribute to the limiting existing body of literature about diet and prevention of weight gain in intensive insulin therapy. The hypotheses tested in this study identified that the effects of an ADA diet and MUFA diet on weight gain, glycemia control, lipid control, blood pressure levels, insulin dose and hypoglycemia events were not statically significant. Possible reasons why there were no significant results was the groups may have differed more than expected, for example, the MUFA group was higher body weight and lab values compared to the ADA group. Also, the groups were older ( $\sim 60$ years of age) and may not have been ready or motivated to make modifications in their diet and daily routine. Many participants were starting insulin as a last measure in controlling their diabetes and many were experiencing diabetes complications as a result of chronic diabetes. There were at least 30 participants in each arm of the study which was enough power to determine significance; however, a larger study sample would gain strength. The study concluded that a diet rich in MUFA can be used effectively to treat weight gain in newly started insulin dependent patients. A major finding from this study was that restriction of the diet to 1600 calories per day in patients, who were accustomed to 2000 calories a day, decreased their A1C value by 1.4 points without weight gain and without extra insulin required. This is clinically significant for providers managing patients starting intensive insulin therapy. Treatment plans with the dietitian need to include frequent individualized training on how to prevent and treat hypoglycemia when restricting calories. Results from this study indicated that changes in ones hunger level, exercise level, and compliance with the diet can effect changes in individuals' weight and metabolic blood values. In conclusion the total calorie count was more important for preventing weight gain and reducing the $\mathrm{A} 1 \mathrm{C}$ in patients on intensive insulin therapy than was dietary composition.

\section{AUTHOR INFORMATON}

Dr. Roland Villareal is the Research Compliance Officer for the Veterans Affairs Southern Nevada Healthcare System (VASNHS) and Lead Faculty Area Chair for the School of Health Administration at the University of Phoenix, Las Vegas campus. Dr. Villareal functioned as Registered Nurse Diabetes Educator and Specialty Care Program Coordinator during this study. IRB approval was obtained through the VA Loma Linda Healthcare System at Loma Linda, California, IRB of record. This material is the result of work supported with resources and the use of facilities at the VASNHS. E-mail: rjv1956@cox.net

\section{REFERENCES}

Bonow, R. O. \& Eckel, R. H. (2003). Diet, obesity, and cardiovascular risk. New England Journal of Medicine, 348 (21), 2057-58.

Flegal, K. M., Carroll, M. D., Ogden, C. L., \& Johnson, C. L. (2002). Prevalence and trends in obesity among US adults. Journal of the American Medical Association, 2002 (288), 1723-27.

Flyn, G. \& Colquhoun, D. (2004). Successful long-term weight loss with a Mediterranean style diet in a primary care medical centre. Asia Pac J Clin Nutr13, S139.

Hu, G., Lindstrom, J., Valle, T. T., Eriksson, J. G., Jousilahti, P., Silventoinen, K., Qiao, Q., \& Tuomilehto, J. (2004). Physical activity, body mass index, and risk of type 2 diabetes in patients with normal or impaired glucose regulation. Archives of Internal Medicine, 164 (8), 892-6.

Kopeland, P. G. \& Grace, C. (2004). New thoughts on managing obesity. GUT, 2004 (53), 1044-1053.

Kris-Etherton, P. M. (1999). Monounsaturated fatty acids and risk of cardiovascular disease. Circulation, 1999 (100), 1253-58.

Marks, J. B. (2004). Advances in obesity treatment: Clinical highlights from the NAASO 2003 annual meeting. Clinical Diabetes, 22 (1), 23-26.

Mayfield, J. A. \& White, R. D. (2004). Insulin therapy for type 2 diabetes: Rescue, augmentation, and replacement of beta-cell function. American Family Physician, 70 (3), 489-500.

McManus, K., Antinoro, L., \& Sacks, F. (2001). A randomized controlled trial of moderate-fat, low-energy diet compared with a low fat, low-energy diet for weight loss in overweight adults. International Journal of Obesity, 2001 (25), 1503-11.

Reaven, G. M. (1988). Banting Lecture 1988. Role of insulin in human disease. Diabetes, 1988 (37), 1595-607. 
Ros, E. (2003). Dietary cis-monounsaturated fatty acids and metabolic control in type 2 diabetes. American Journal of Clinical Nutrition, 78 (suppl), 617S-25S.

Wijenaike, N. (2004). Living with diabetes. West Suffolk Diabetes Service. On Line. Retrieved from http://www.diabetes- information.co.uk/Living\%20with\%20Diabetes/Insulin\%20tre... 